
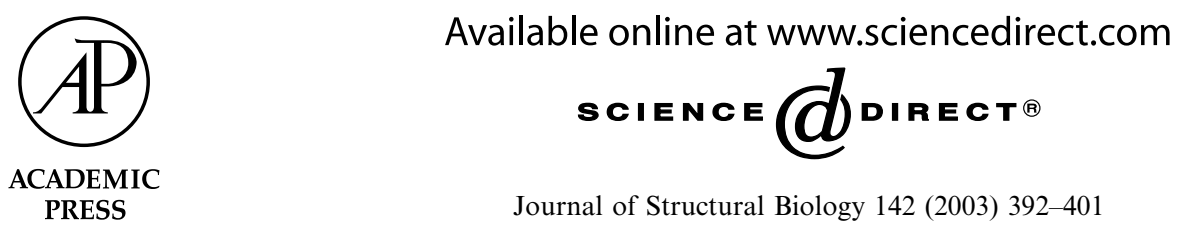

Journal of Structural Biology 142 (2003) 392-401

\title{
Automatic CTF correction for single particles based upon multivariate statistical analysis of individual power spectra
}

\author{
B. Sander, M.M. Golas, and H. Stark* \\ Max Planck Institute for Biophysical Chemistry, Am Fassberg 11, 37077 Göttingen, Germany
}

Received 19 December 2002, and in revised form 28 February 2003

\begin{abstract}
Three-dimensional electron cryomicroscopy of randomly oriented single particles is a method that is suitable for the determination of three-dimensional structures of macromolecular complexes at molecular resolution. However, the electron-microscopical projection images are modulated by a contrast transfer function (CTF) that prevents the calculation of three-dimensional reconstructions of biological complexes at high resolution from uncorrected images. We describe here an automated method for the accurate determination and correction of the CTF parameters defocus, twofold astigmatism and amplitude-contrast proportion from single-particle images. At the same time, the method allows the frequency-dependent signal decrease ( $B$ factor) and the nonconvoluted background signal to be estimated. The method involves the classification of the power spectra of single-particle images into groups with similar CTF parameters; this is done by multivariate statistical analysis (MSA) and hierarchically ascending classification (HAC). Averaging over several power spectra generates class averages with enhanced signal-to-noise ratios. The correct CTF parameters can be deduced from these class averages by applying an iterative correlation procedure with theoretical CTF functions; they are then used to correct the raw images. Furthermore, the method enables the tilt axis of the sample holder to be determined and allows the elimination of individual poor-quality images that show high drift or charging effects.
\end{abstract}

(c) 2003 Elsevier Science (USA). All rights reserved.

Keywords: Contrast transfer function; CTF; Defocus correction; Electron microscopy

\section{Introduction}

The periodic change of sign of the contrast transfer function (CTF) has far-reaching consequences for the reconstruction of particle structures. The modulation of electron microscopical images by the CTF causes points in the object plane to appear not as points, but as concentric, overlapping rings, with phase reversals in Fourier space that occur with increasing rapidity at higher spatial frequencies. The appearance of CTF is a consequence of the necessary deviation from the Gaussian focal plane into the underfocus in phase-contrast electron microscopy (Scherzer, 1949). Optimal phase contrast cannot be achieved for all diffraction angles and space frequencies simultaneously. In particular, although it is true that a high degree of defocusing (several

\footnotetext{
${ }^{*}$ Corresponding author. Fax: +49(0)551-201-1197.

E-mail address: holger.stark@mpibpc.mpg.de (H. Stark).
}

times the Scherzer focus) leads to an early increase in $\mathrm{CTF}$ and thus to selective emphasis of the lower spatial frequencies, allowing the particles to appear with enhanced contrast, a strong defocus has the attendant drawback of producing a very rapid sign change of the $\mathrm{CTF}$ in the region of high-resolution image information.

Almost all the calculations of three-dimensional (3D) structures solved recently by electron-microscopic methods have therefore included a deconvolution step intended to free the 3-D density from the influence of the $\mathrm{CTF}$ and thus to raise the attainable resolution beyond the first zero-crossing of the CTF. In this context one can distinguish between, on the one hand, the method of focal pairs (Conway and Steven, 1999; Frank and Penczek, 1995), which is based upon pairs of recordings of the same field with different defoci, and, on the other hand, methods that are aimed at extracting the CTF parameters from the power spectra of individual micrographs. There exist various possibilities to improve the visibility of poorly visible phase reversals in power 
spectra (also called Thon rings; (Thon, 1966)). These methods include averaging over large negative regions together with rotational averaging (Zhu et al., 1997), summing of power spectra to enhance the signal-tonoise ratio (Zhou et al., 1996) and periodogram averaging from mutually overlapping regions (Fernandez et al., 1997). Various mathematical methods have been applied successfully to the extraction of the CTF parameters from Thon-ring images. These include leastsquares fitting (Zhu et al., 1997), graphical user interfaces (Ludtke et al., 1999; Zhou et al., 1996), and the fitting used here, which is based upon the correlation coefficient between the estimated power spectral density and theoretical, two-dimensional (2-D) CTF functions (Radermacher et al., 2001; Tani et al., 1996).

The method described here for the correction of defocus and astigmatism, functions precisely and rapidly for large data sets of several thousand single particles and has, in addition to a high degree of automation, the advantage that the exact position of the particle in the micrograph does not need to be known. It is based upon the diffuse signal of the supporting amorphous carbon film onto which the particles are adsorbed, and it employs the MSA classification implemented by software packages such as IMAGIC (van Heel et al., 1996).

\subsection{Physical foundations}

Let $\Delta z$ be the defocus and $C_{\mathrm{s}}$ the spherical aberration of the electromagnetic lens, and $q$ the spatial frequency defined as the quotient of the diffraction angle and the wavelength $\lambda$. The phase shift $W(q)$ in the representation of a pure phase object is then given by

$W(q)=\frac{\pi}{2}\left(C_{\mathrm{s}} \lambda^{3} q^{4}-2 \Delta z \lambda q^{2}\right)$.

The phase-contrast transfer function $B(q)$ can be derived from this equation; its sign is chosen such that $B(q)>0$ for positive phase contrast (Scherzer, 1949; Thon, 1966)

$$
\begin{aligned}
B(q) & =-2 \sin W(q) \\
& =-2 \cdot \sin \left[\frac{\pi}{2}\left(C_{\mathrm{s}} \lambda^{3} q^{4}-2 \Delta z \lambda q^{2}\right)\right] .
\end{aligned}
$$

The contrast in the representation of biological particles can be described with good approximation by this phase-contrast transfer function. However, especially in the region of low and medium spatial frequencies, a shift in the zero-crossings of the measured CTF relative to the theoretical phase-CTF can be observed. This has its origin in the absorption amplitude contrast.

A good approximation of the combined effect of phase and amplitude contrast in the generation of images is achieved - on the assumption of an amplitudecontrast proportion that is independent of the spatial frequency (Erickson and Klug, 1971; Toyoshima et al.,
1993) - by adding a cosine term to Eq. (2). If $F$ is the amplitude-contrast proportion, then the combined CTF $H(q)$ is obtained

$H(q)=-2 \cdot[(1-F) \cdot \sin W(q)+F \cdot \cos W(q)]$.

However, under realistic conditions of measurement a simple, radially symmetric CTF cannot be assumed, since in practice astigmatism cannot be completely corrected for experimentally. Twofold astigmatism leads to a superposition of two CTFs with two (different) defocus values $\Delta z_{x}$ and $\Delta z_{y}$ along the two orthogonal principal axes. Rotational averaging of power spectra, which in many cases is a first step in the determination of the CTF parameters, prevents the determination of the astigmatism parameters. However, if the signal-tonoise ratio is obtained from averaging similar power spectra applying MSA and subsequent classification, then the astigmatism information is preserved. The astigmatism parameters can then be determined and corrected for.

A further factor that must be taken into consideration is the spatial-frequency-dependent reduction in signal strength; this has several causes and is summarised in the so-called experimental $B$ factor. Contributions to this exponential decay in the CTF maxima come from aberrations in the microscope, the incoherence of the electron source (Frank, 1973; Wade and Frank, 1977), the instability of the sample holder in the microscope, the modulation transfer function (MTF) of the film material used and the MTF of the scanner used (Saad et al., 2001). The influence of various resolutionlimiting factors can be summarised as an exponential factor $\mathrm{e}^{-2 B q^{2}}$, where $B$ is the $B$ factor (Glaeser and Downing, 1992; Saad et al., 2001). Furthermore, an additive frequency-dependent background noise term $S(q)$ that is not convoluted by the CTF must be considered; this arises from various sources such as noise in the optical media and non-elastic scattering of electrons. The background noise can be described by the exponential decay of the absolute positions of the $\mathrm{CTF}^{2}$ minima and the noise function can be approximated by a Gaussian profile with three unknown parameters ( $\mathrm{Zhu}$ et al., 1997). In contrast to the exponential $B$ factor that describes the amplitude decay of the maxima in the $\mathrm{CTF}^{2}$, the background noise function explains the exponential decay of the values of the $\mathrm{CTF}^{2}$ minima in the power spectrum.

Since the method to be described employs a correlation-based fitting that is not influenced by the absolute values in the power spectra and the theoretical referenceCTFs, the rotational average of a Gaussian profile with two unknown variables $(a, c)$ gives a good representation of the non-convoluted background noise to improve the correlation based fitting procedure:

$S(q)=a \cdot \mathrm{e}^{-q / c^{2}}$. 
Thus, all in all, the contrast to be expected is expressed by

$$
\begin{aligned}
H\left(q_{x y}\right)= & -2 \cdot \mathrm{e}^{-2 B q^{2}}\left[(1-F) \cdot \sin W\left(q_{x y}\right)\right. \\
& \left.+F \cdot \cos W\left(q_{x y}\right)\right]+S(q) .
\end{aligned}
$$

\section{Methods}

\subsection{Preparation and measurement}

To test the software, two different samples (70S ribosomes and U1 snRNPs) were prepared by three different methods (native cryo-preparation in holes, native cryo-preparation on carbon film, and cryo-negativestaining) and were examined in three different Philips electron microscopes (SOPHIE, CM200FEG, and CM120). For the cryo-negative-stain method, 70S ribosomes were adsorbed onto a thin carbon film, stained with $2 \%$ uranyl formate and covered with a second carbon film ["sandwich" method (Kastner, 1998; Tischendorf et al., 1974; Valentine et al., 1968)]. The preparations were frozen in liquid nitrogen and images were obtained in the electron microscope under lowdose cryo-conditions using electron dosages of 15-20 electrons $/ \AA^{2}$. After selection of particles with the software IMAGIC-5, the particle images were extracted from the digitised negative, and the CTF parameters were corrected locally, at the level of single particles. To obtain high-resolution information in the corrected images, it was necessary (on account of the point spreading) to use a sufficiently large window when the single-particle images were being extracted for defocus correction; the required size of the pixel window generally depends upon the defocus value and was in our case about twice the size of the maximum particle diameter.

To test the applicability of the method for "native cryo" preparations and to compare amplitude-contrast values between cryo-negative-stain samples and native cryo-conditions (Adrian et al., 1984), three different additional "native cryo" samples were prepared. First of all, U1 snRNP particles in vitreous ice (Stark et al., 2001) were prepared by shock-freezing in liquid ethane. The concentration of U1 snRNP was high enough to cover the entire ice hole with a lawn of protein-RNA particles, so that a signal of adequate strength for iterative analysis was obtained. Second, native $70 \mathrm{~S}$ ribosomes were prepared in vitreous ice on a carbon support film and the images thus obtained were used for comparative measurement of the amplitude-contrast proportion. Third, native $70 \mathrm{~S}$ ribosomes were also prepared in vitreous ice on a holey carbon grid. The different samples were imaged in various microscopes to test the applicability of our method for a wide range of different sample preparation techniques and to allow comparison of the $B$ factors measured. The microscopes used were (i) a Philips CM200 with an FEG cathode and twin lens $\left(C_{\mathrm{s}}=2.0 \mathrm{~mm}\right)$ operated at $200 \mathrm{kV}$ with a Gatan cryoholder, (ii) a Philips CM120 $\left(C_{\mathrm{s}}=6.3 \mathrm{~mm}\right)$ with a LaB6 cathode and BioTwin lens operated at $120 \mathrm{kV}$ with a Gatan cryoholder, and (iii) SOPHIE, a modified Philips CM20 FEG, equipped with a helium-cooled superconducting objective lens $\left(C_{\mathrm{s}}=1.35 \mathrm{~mm}\right)$ (Zemlin et al., 1994) and operated at $200 \mathrm{kV}$.

\subsection{Classification of power spectra according to CTF parameters}

For correction, data sets of selected single particles with typically several thousand individual images are used. Multivariate statistical analysis (MSA) (Frank and Van Heel, 1982; Lebart et al., 1984; van Heel, 1984) is carried out on the power spectra of these images (Fig. 1). The prerequisite for optimal results is a conventional high-pass filtering of the images with an inverse Gaussian profile for the computation of the power spectra, in order to suppress the large signal amplitude at the centre of the power spectrum and at the same time to raise the relative signal strength in the regions of rapid CTF sign change. In the digitisation of the micrographs, very small sampling increments are generally used, in order to exploit the scanner's resolution as far as possible. Thus, the signal in the power spectra approaches zero long before the Nyquist frequency is reached (oversampling). The procedure described here is greatly accelerated by storing only the central regions of the computed power spectra and using these to perform the MSA. In the MSA, 10-20 eigenimages are computed and a classification (HAC) (van Heel, 1984) of the 2-D power spectrum is carried out, so that some $20-50$ individual power spectra with similar CTF parameters are placed in each class. If a data set contains astigmatic images, then a characteristic phenomenon can be observed, as in the example shown: some eigenimages display characteristic displaced semi-circles (see eigenimage No. 8 in Fig. 2A).

\subsection{Iterative determination of the CTF parameters}

The class averages of power spectra thus obtained from individual molecular images, with almost identical CTF parameters, are used to determine iteratively the fundamental parameters defocus, amplitude-contrast proportion, astigmatism, $B$ factor and the non-convoluted background noise. The parameters defocus, amplitude contrast and astigmatism are then used for correction of the single images already classified according to the similarities found in their power spectra. The basis of the iterative procedure is the computation of theoretical power spectra over a presumed interval and the calculation of the correlation coefficient with the measured power spectra, as already described (Rad- 


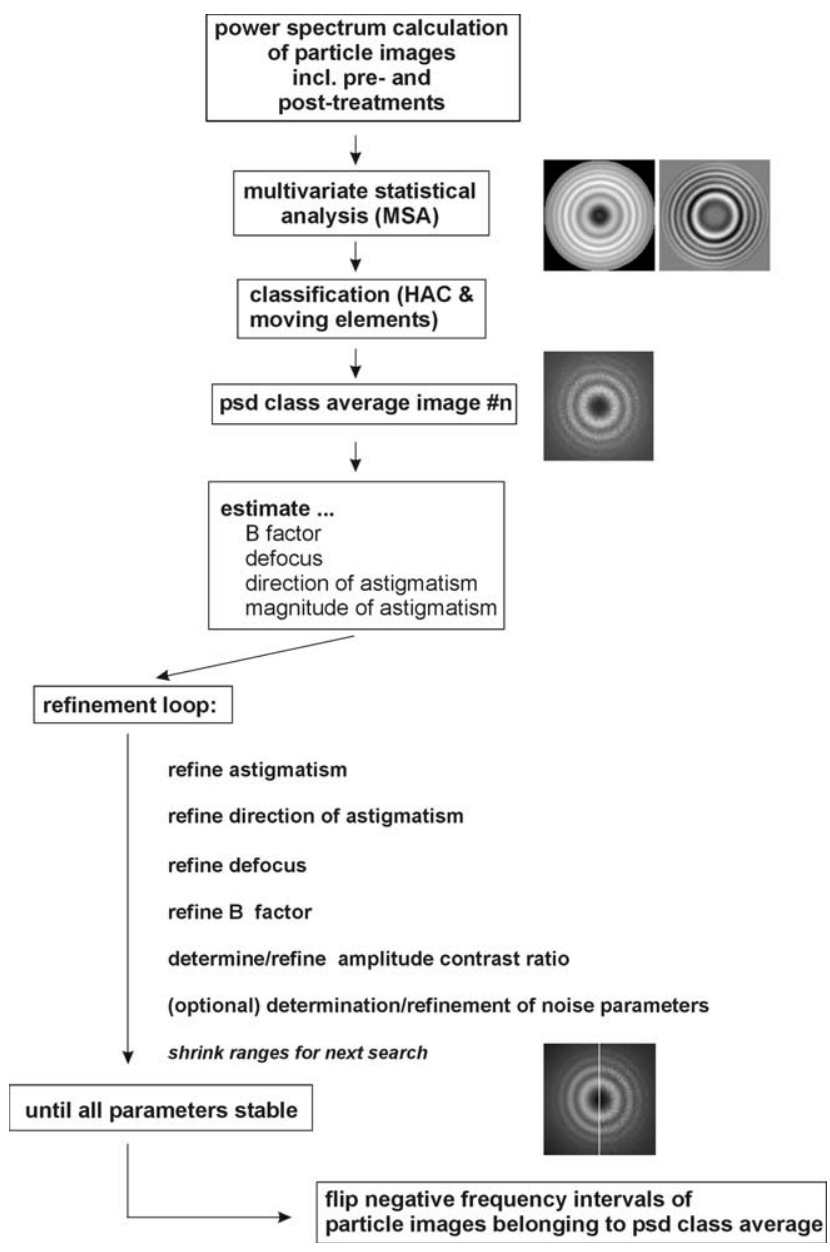

Fig. 1. Flow-chart for defocus correction. High-pass-filtered and excised power spectra are subjected to multivariate statistical analysis (MSA), whereby the properties of the Thon rings are obtained as principal components of the data set. The class averages obtained from the classification with enhanced signal-to-noise ratios are used in the iterative determination of the parameters defocus, astigmatism, $B$ factor, amplitude-contrast proportion and a noise function on the basis of the correlation coefficient with theoretical CTF functions. The parameters defocus and astigmatism, found in this way, are then used for correction of the single-particle images in each class.

ermacher et al., 2001; Tani et al., 1996). Various orders for iterative fitting of the parameters have been tried out on theoretical 2-D power spectra with various defocus and astigmatism values, the goal being to find a strictly converging algorithm. In the procedure described here, one parameter at a time is varied, and the value of this parameter corresponding to the correlation maximum found is stored and used in subsequent fitting until a value that correlates more closely with the class average is found. Because of the point symmetry of power spectra, it is possible to accelerate this process by conducting the calculations on one half of the image only.

In principle, systematic errors can prevent the convergence of the iterative fitting to correlation maxima. These errors include especially the neglect of all effects that lead to intensity gradients between the centre and the edge, such as the $B$ factor and, to a lesser extent, nonconvoluted background noise. The procedure shown in Fig. 1 therefore begins with preliminary estimation of the $B$ factor by fitting to decreasing 2-D exponential functions with varying full width at half maximum. There follows a first search for the defocus at intervals of $50 \AA$ in a region defined by the user. The correlation of the Thon-ring images with theoretical CTF references can-according to signal strength-lead to periodical secondary maxima, as is observed in interference phenomena. The amplitude of these secondary maxima is lowered by initial high-pass filtering of the class average, so that an unambiguous determination of the principal maximum becomes possible (not shown). The course of the correlation coefficient as a function of the defocus used to calculate the reference $\mathrm{CTF}$, with a typical peak in the height of the defocus sought, is shown in Fig. 3. At high twofold astigmatism values, even correlation curves with two principal peaks separated by a trough can arise; these express different defocus values in the two principal directions. In this case, the local minimum between these maxima is automatically chosen as a good first estimate of the underlying defocus. To determine the direction of the astigmatism it is not necessary to know its exact magnitude. For the first iteration we assume an astigmatism of $50 \AA$ and vary the direction in steps of $6^{\circ}$. The direction of the astigmatism thus found is then used to find the magnitude of the astigmatism. All parameters are then refined sequentially, as shown in Fig. 1, whereby both the step size is reduced and the search region is systematically restricted, in order to accelerate the search; if the data inserted by the user were incorrect, then the search region can also be moved. In the generation of the theoretical CTF functions the high-pass filter, with which the single-particle images were pretreated, is also taken into account (Fig. 4). Once all the parameters have reached stable values, the individual images, which were classified into corresponding class averages, are corrected. The success of the iteration can immediately be judged, as at the end of processing each class average, our program stores an image with the measured and (opposite to it) the fitted CTF; in this image, the Thon rings should fit one another. A gallery of individual power spectra, class averages and the fitted rings at various defocus and astigmatism values is shown in Fig. 2. The correlation coefficients obtained are, dependent on the number of single images and the signal strength in the data set, usually in the region of $60-95 \%$. The general applicability and precision of the CTF parameter-determination method were evaluated by using test images with varying signal-to-noise ratios and $B$ factors. The high $B$ factors used in the test make highresolution Thon rings invisible. However, it turned out that the accuracy of the CTF parameter determination was not strongly dependent on the visibility of highresolution Thon rings. The method also appeared quite 
A

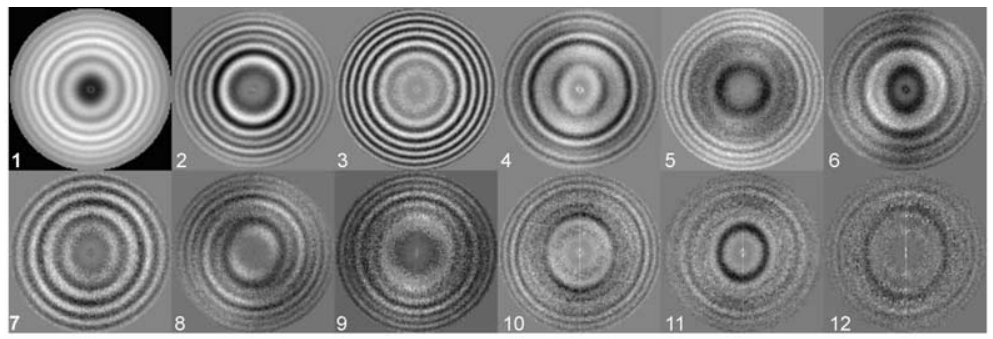

B

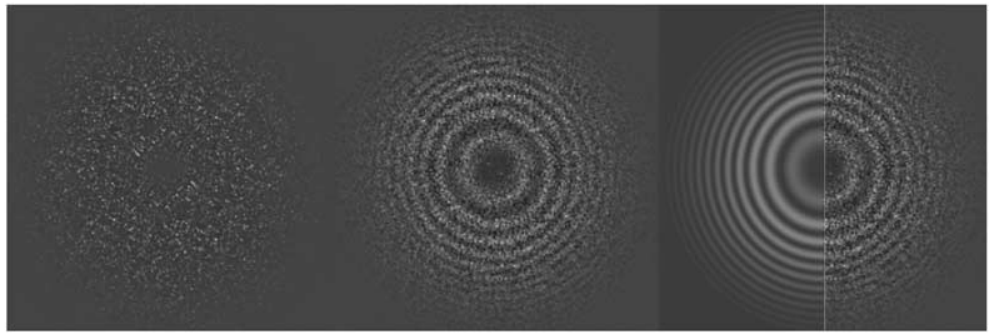

C

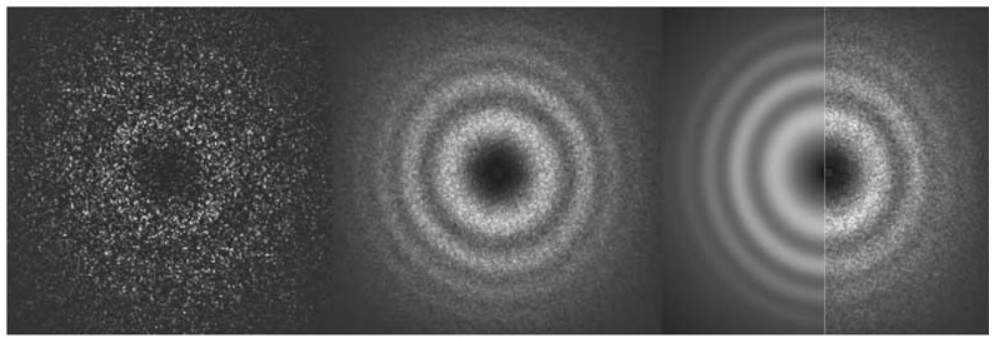

D

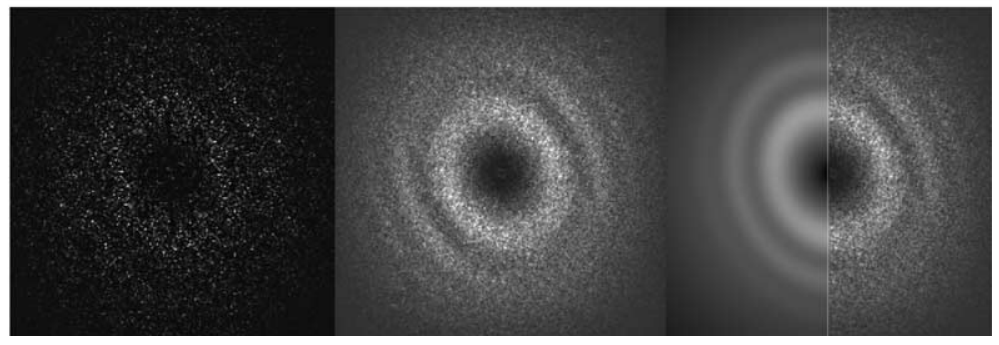

Fig. 2. Multivariate statistical analysis (MSA) of power spectra. (A) Twelve eigenimages from the MSA of a typical data set of power spectra (size of the particle images, $1024 \times 1024$ pixels, corresponding to approximately $80 \times 80 \mathrm{~nm}$ in the sample; size of power spectra, $256 \times 256$ pixel). (B) Left, individual power spectrum; centre, class average of a class containing 50 images; right, visualisation of the fit. Each picture on the right contains the result in the left-hand half and the class average in the right-hand half. On a PC with AMD MP1800+ CPU running under Windows 2000, a typical calculation of the CTF parameters in the $256 \times 256$-pixel frame takes about 3 min for each spectrum to be corrected. (C) As in (B) in a case where astigmatism appeared. The class average shown represents 57 images. (D) As in (B) with drift of the sample cryo-holder and directionally truncated Thon rings. The class average shown represents 42 images.

robust with respect to variations of the SNR in the range of $10-0.1$, with relative errors in the range $0.2-1.5 \%$ for the defocus determination and in the range of $5-10 \%$ for the astigmatism determination. (For an experimental approach to determining the accuracy of the fitting procedure in combination with the MSA of power spectra, see below.)

\section{Results and discussion}

\subsection{Accuracy of the method}

The general strategy for finding local CTF parameters rests upon the retrospective estimation of the local power-spectral density, by means of which the square of the CTF can be made visible. The CTF parameters at a position on the image are analysed by Fourier-transforming extracted images of individual macromolecules and squaring the result. The smaller the pixel frame used, the more accurately it represents local conditions. At the same time, the signal-to-noise ratio at the level of the individual power spectrum becomes increasingly poor, and the local $\mathrm{FFT}^{2}$ (squared Fourier transform) results in a correspondingly worsening estimate of the underlying power-spectral density.

For this reason, in procedures for defocus correction that are based upon the analysis of local power spectra, the attainable accuracy should be correlated with the size of the pixel frame: the larger the pixel 


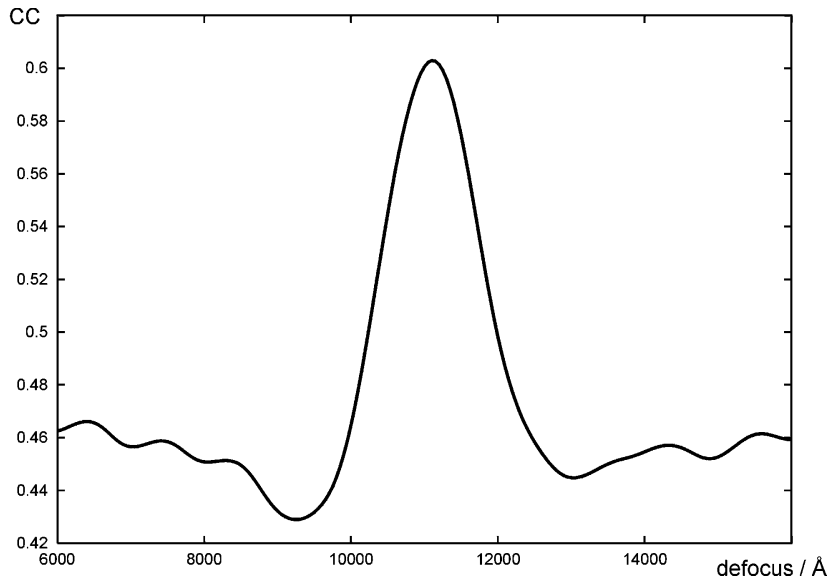

Fig. 3. Correlation coefficients between theoretically calculated power spectra in the range of $6000-15,000 \AA$ and the measured power spectrum of a class average with a defocus of approximately $11,000 \AA$. The defocus sought is revealed by a clear maximum, implying that the best possible correspondence between the class average and the powerspectral density reference is found at this point.

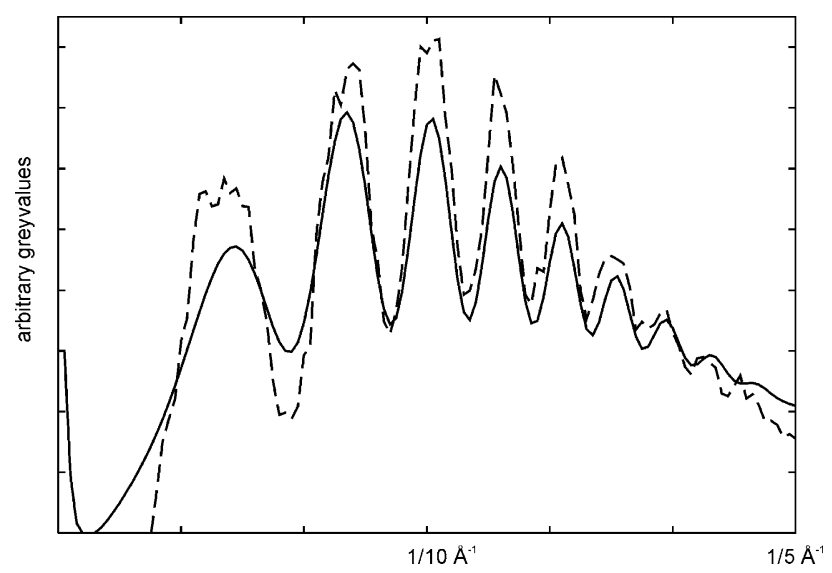

Fig. 4. Quality of a theoretical CTF fitted to a power spectrum class average. The rotationally averaged power spectral density (arbitrary grey values) was plotted as a function of the spatial frequency. Dashed line: 1-D plot of a rotationally averaged class average. Continuous line: 1-D plot of the theoretical CTF found for this class average. There is good correspondence between the calculated and measured functions with respect to the location of zero-crossings as well as to the estimation of the background noise. The first maximum has a smaller amplitude than the following maxima, in consequence of the high-pass filtering of the single-particle images.

frame, the better the signal-to-noise ratio in the power spectra-that is, the visibility of the Thon rings-becomes. However, if very large image excerpts or even the entire micrograph are used, then local differences in the CTF-arising, for example, through tilting of the sample - cannot be taken into account. Generally, the same is true for "native" cryo-preparations of individual macromolecules (here, ribosomes) embedded in vitreous ice in the holes on a perforated carbon film. In such cases, the signal in the power spectra is very weak, so that MSA analysis and classification of these power spectra usually fail. However, the method still works in some special cases where either the concentration of molecules observed in the ice holes (simulated here by the lawn of U1 snRNP) is very high and/ or macromolecular complexes with high molecular weight-such as icosahedral viruses - are imaged. In all other cases, the method cannot be used to determine the "local" CTF parameters of each macromolecule. Instead, however, the automatic fitting procedure can be applied to the average of overlapping power spectra from an entire micrograph, and the resulting "global" CTF parameters can then be applied to correct all molecular images of a single micrograph using the same CTF values. The accuracy of this global CTF parameter determination can be much improved by prior calibration of the zero-tilt position of the holder in the electron microscope (see below). Our method can be used to determine this zero tilt when it is applied to images that give an additional carbon-film signal.

To investigate the defocus variance between neighbouring locations on micrographs with carbon support film as a function of pixel size, a micrograph measuring $9 \times 12 \mathrm{~cm}$ was taken $(50,000$-fold enlargement, digitisation with $4 \mu \mathrm{m}$, i.e., $0.8 \AA$ per pixel at the level of the sample) and about 21,000 points were distributed on it at a uniform spacing of 128 pixels. Overlapping image areas with variously sized pixel frames were excised. Pixel frames of sizes $256 \times 256,384 \times 384,512 \times 512$, $640 \times 640$, and $800 \times 800$ pixels were used. The sizes of the power spectra after excision of the central region were $80 \times 80,128 \times 128,160 \times 160,200 \times 200$, and $256 \times 256$ pixels, each of which corresponded to a critical Nyquist frequency of about $(5 \AA)^{-1}$. The method described here was applied to this data set and the dependence of the defocus found upon the position on the negative was represented in a three-dimensional plot (Fig. 5). The thickness of the layer of neighbouring measurement points was used as a measure of the accuracy in determining the defocus attainable with a given pixel frame. While small image excerpts of $256 \times 256$ pixels $(20 \times 20 \mathrm{~nm}$ at sample level $)$ cause high error scatters of more than $900 \AA$, a substantial reduction in the scatter of neighbouring measurement points is possible with larger excerpts; for the largest pixel frames examined here $(800 \times 800$ pixels; $64 \times 64 \mathrm{~nm}$ at sample level) this lay below $200 \AA$. The cryo-negativestain preparation of $70 \mathrm{~S}$ ribosomes in a sandwich of ribosome-stain-water mixture between two carbon films used in the experiments has an estimated thickness of approximately $350 \AA(2 \times 50 \AA$ carbon plus $250 \AA$ ribosomes). The maximum accuracy of the defocus determination of $200 \AA$ leads to the conclusion that the program detects an average defocus between the upper and lower carbon film layers. The defocus value determined for the particle thus lies somewhere in the central 


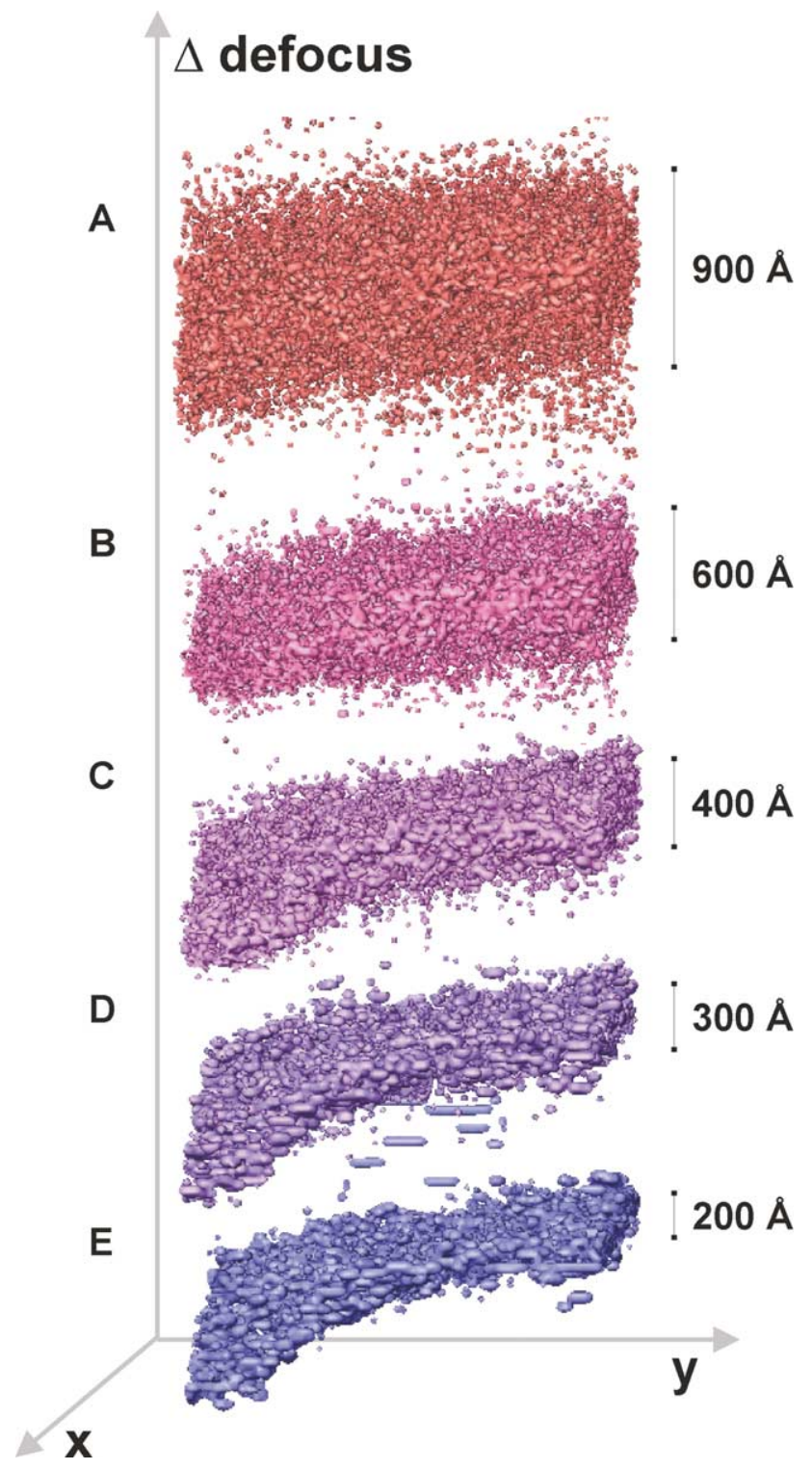

Fig. 5. Measurement of the accuracy to be expected in the defocus method, as a function of the size of the pixel window used in the calculation of power spectra. Measured defocus values of closely adjacent points are shown in a three-dimensional plot as a function of $x, y$-position in the micrograph. As enlarging the pixel frame results in an improved estimate of the underlying power-spectral density, the scatter of the measured defocus values of the points in the micrograph decreases correspondingly. (A) $256 \times 256$-pixel sections of the micrograph $(20 \mathrm{~nm} \times 20 \mathrm{~nm}$ on the scale of the sample). (B) $384 \times 384$-pixel sections $(30 \mathrm{~nm} \times 30 \mathrm{~nm})$ with a scatter of $600 \AA$. (C) $512 \times 512$-pixel sections $(40 \mathrm{~nm} \times 40 \mathrm{~nm})$. (D) $640 \times 640$-pixel sections $(51 \mathrm{~nm} \times 51 \mathrm{~nm})$. (E) $800 \times 800$-pixel sections $(64 \mathrm{~nm} \times 64 \mathrm{~nm})$. In (A) the scatter is more than $900 \AA$, and the tilt of the sample can hardly be seen. With the largest pixel frame used here, $800 \times 800$ pixels, the scatter resulting from the defocus values of closely adjacent neighbours is approximately $200 \AA$. Besides the deviation of the cryo-holder (about $3^{\circ}$ ) from the zero position, the foremost left-hand edge indicates an additional bending of the sample leading to an overall measured defocus gradient of $\sim 1000 \AA$ in the micrograph. region of the sandwich and therefore intersects a plane through the molecule.

Remarkably, the narrow scattering of the results allows the mounting of the preparation, including tilt of the sample holder and local distortion, to be visualised directly. In Fig. 5E an overall tilt of about $3^{\circ}$ is seen, and this is ascribed to inaccurate calibration of the zero position of the holder [see also (van Heel et al., 2000)]. Furthermore, a bending of the foremost, left-hand edge can be seen. Especially with preparations on carbon film, bends and folds of this kind can be expected, because of the manipulation of the material with tweezers. For the sample-holder we use, the difference in focus that arises through the tilt of the sample is $\sim 1000 \AA$ and is thus very significant for the CTF correction in highresolution investigations. Alternatively, the tilt angle of the holder can be measured and reduced to zero by suitable calibration. However, a slight tilt can also be desirable for high-resolution 3-D structure determination, as in this way the defocus multiplicity in the data set is raised and the zero-crossings in the CTF can thus be compensated for more easily.

\subsection{Dependence of the $B$ factor upon the conditions of measurement}

The consideration of the $B$ factor in the iterative calculation of CTF parameters in the present work is especially important because - as has been shown by tests with theoretically calculated power spectra-it allows a continuous convergence of the algorithm without

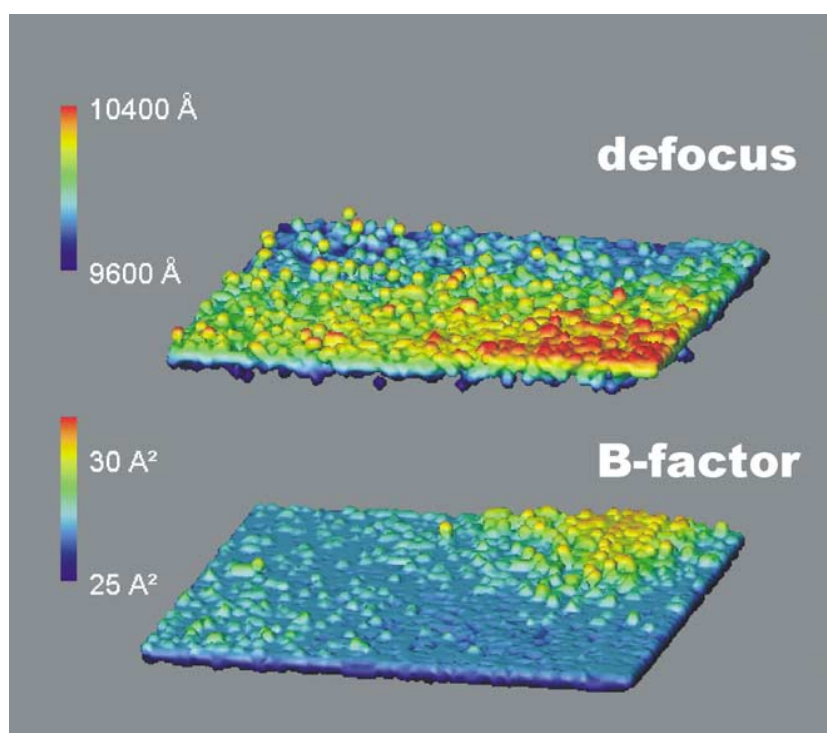

Fig. 6. Variations in defocus and $B$ factor as functions of the $x, y$-position in the micrograph. In the zero position of the holder, the residual deviation results in a tilt of about $3^{\circ}$. In addition, a characteristic distribution of measured $B$ factors with a maximum in the right-hand hindmost region of the micrograph, corresponding to a locally reduced quality, is shown. 
the appearance of systematic errors due to deviation of the measured power spectra near the Nyquist frequency in the theoretical CTF references. A similar effect could also arise through the use of a weighting function in the calculation of the correlation coefficient that takes less full account of the high-frequency regions in the power spectra (Tani et al., 1996). However, accurate knowledge of the experimental $B$ factor can in several ways be useful for the computation of the 3-D structure of the particle: a correction of the $B$ factor at the level of the individual particle, the class averages or the 3-D density is necessary for the visualisation of highly resolved structural information (Bottcher et al., 1997). However, correction of the $B$ factor immediately after particle selection, before the actual structure determination, seems to us to be disadvantageous, for two reasons. First, the $B$ factor correction at the level of individual particles may lead to a signal-to-noise ratio that is too low for many of the algorithms in use in image-processing, resulting in extremely poor particle visibility and a very low image contrast, on account of the exponential increase in high-frequency noise at high spatial frequencies. Second, an upper frequency limit is necessary for $B$ factor correction of single-particle images, after which the correction can no longer be applied, as excessive noise amplification must be avoided. This frequency limit should ideally correspond to the resolution to be attained in the 3-D reconstruction and is therefore not known a priori. A $B$ factor correction at the single-particle level can thus only be meaningfully carried out after a stable level of resolution has been reached, corresponding to the highest resolution attainable with the data set.

Furthermore, the knowledge of local $B$ factors in the micrograph can be utilised in estimating the image quality as a function of the position of a particle (Gao et al., 2002). The $B$ factors of a typical micrograph are plotted as a function of position in Fig. 6. A wedgeshaped zone with increased $B$ factors is seen; this may be due to local charging effects during flood beam microscopy.

It was not the aim of this study to measure systematically $B$ factors under many different conditions using the methods described here. Nevertheless, we describe some significant results obtained on negatively contrasted preparations under cryo-conditions (see also Table 1) and at typical defocus values between 0.5 and $1 \mu \mathrm{m}$, where the $B$ factor can be regarded as being lar-

Table 1

$B$ factors (cryo-negative-stain, double carbon film) as measured with different microscopes

\begin{tabular}{ll}
\hline Microscope & $B$ factor $\left(\AA^{2}\right)$ \\
\hline CM 120-LAB6, 120 kV & $80 \pm 5$ \\
CM 200-FEG, 200 kV & $28 \pm 2$ \\
SOPHIE-FEG, 200 kV & $17 \pm 1$ \\
\hline
\end{tabular}

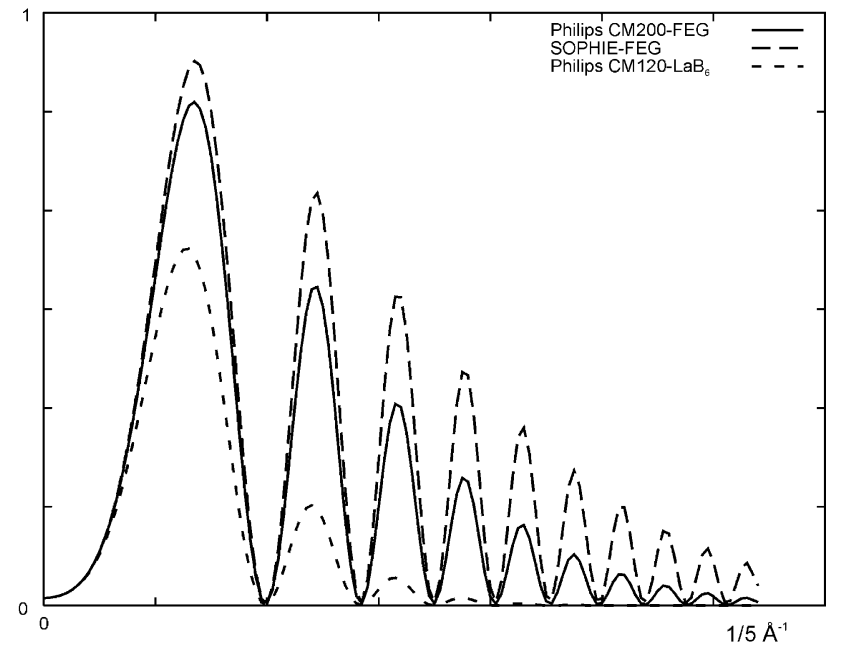

Fig. 7. Microscope-dependent signal fall-off as a function of spatial frequencies using $B$ factors calculated from cryo-negative-stain micrographs of $70 \mathrm{~S}$ ribosomes in $2 \%$ uranyl formate (sandwich method). Values of $B$ factors obtained were: In the Philips CM120 microscope, $80 \AA^{2}$; in the Philips CM200-FEG, $28 \AA^{2}$; in the helium-cooled, superconducting objective lens of the microscope SOPHIE, approximately $17 \AA^{2}$.

gely independent of the defocus (Saad et al., 2001). The strong dependence of the $B$ factor upon the coherence of the electron source is known (Zemlin, 1994; Hewat and Neumann, 2002). With the Philips CM200 electron microscope (Twin-Lens, $C_{\mathrm{s}}=2.0 \mathrm{~mm}$ ) and FEG-cathode, we measured a $B$ factor of approximately $28 \AA^{2}$ in typical micrographs. The value is thus much smaller than those described elsewhere, viz., $50-150 \AA^{2}$ (see also (Saad et al., 2001)). The small value appears to be due to the use of stain together with the double carbon film, as a comparable preparation of $70 \mathrm{~S}$ ribosomes on a single carbon film without stain gave a $B$ factor of about $85 \AA^{2}$. Another significant quantity is the ratio of the $B$ factors obtained with a conventional liquid nitrogencooled electron microscope and those obtained with the liquid helium-cooled electron microscope SOPHIE $\left(17 \AA^{2}\right)$ (Zemlin et al., 1994), which was found to be about 1.6 when the techniques described were used. This significant difference shows that the use of heliumcooled microscopes may come to play a substantial part in improving the resolution of 3-D structures (Fig. 7).

\subsection{Dependence of the amplitude-contrast proportion upon the preparation method}

The amplitude-contrast proportion leads to a shift of the Thon rings (especially the more central ones) in the direction of low spatial frequencies and thus to unambiguous changes compared with pure phase CTF. The mean amplitude-contrast proportions found under three different sets of preparation conditions are shown in Table 2. For a native cryo preparation of RNA-protein 
Table 2

Amplitude-contrast proportions (Philips CM200, FEG-cathode) as measured for different preparation methods

\begin{tabular}{ll}
\hline Preparation method & $\begin{array}{l}\text { Amplitude-contrast } \\
\text { proportion (\%) }\end{array}$ \\
\hline Cryo-negative-staining (sandwich) & $14 \pm 3$ \\
Native cryo-preparation on carbon film & $9 \pm 4$ \\
Native cryo-preparation in vitreous ice & $6 \pm 2$ \\
\hline
\end{tabular}

particles (human U1 snRNP particles) without stain or support film we obtained an amplitude-contrast proportion of approximately $6 \%$. For a native preparation of $70 \mathrm{~S}$ ribosomes on carbon film we found an amplitude-contrast proportion of $9 \%$. The amplitude-contrast proportion for the cryo-negative-stain preparation was, however, $14 \%$, and thus about twice as large as in native cryo preparations (see Table 2). The values found here are in good agreement with values determined by other methods (Toyoshima and Unwin, 1988; Toyoshima et al., 1993; Zhu et al., 1997).

\section{Conclusions}

In the imaging of biological particles on thin carbon films, the signal of the object is superposed upon a homogeneous signal from the amorphous carbon, the suitability of which for the estimation of the underlying power-spectral density and thus for the CTF correction is already well documented. In the determination of the exact CTF parameters at the position of the imaged particle, it should not necessarily be assumed that the CTF is constant in the entire negative. Rather, the individual parameters may vary owing to a tilt of the sample holder, to local charging or to distortion of the sample. For this reason, multivariate statistical analysis (MSA) is ideally suited not only to the identification of different views of individual particle images, but also to the analysis of the power spectra, especially as power spectra are in themselves optimally centred. The classification implemented in IMAGIC-5 (HAC plus Moving Elements Refinement) (Van Heel, 1984) is able to classify power spectra into categories of very similar CTF parameter values. Surprisingly, this allows not only the defocus, but also other parameters, in particular astigmatism, to be distinguished without the need for rotational averaging. Under the right conditions this even becomes visible in the MSA in characteristic eigenimages. Averaging of the power spectra grouped into the same classes leads to class averages with Thon rings that, owing to the summation of signals, have become clearly visible. These can be estimated, on the basis of their power spectra, with much greater accuracy than the $\mathrm{FFT}^{2}$ of the individual particle images. The class averages can thus be used for accurate determination of CTF parameters. The multivariate statistical analysis finds-as shown in the eigenimages-mainly the Thon rings as principal properties of the data set. It may therefore be taken that the subsequent classification is really based upon CTF parameters and not upon structure factors. Furthermore, the averaging of many power spectra reduces the contribution of individual particles to the signal in the FFT ${ }^{2}$. Qualitatively poor images, characterised by charging or drift effects, are also identified, as even power spectra with directionally truncated Thon rings are reliably classified by the MSA procedure. The method presented here for local defocus correction has the advantage of fully automated processing of the single-particle images without the need to store the entire raw data after selection of the particles. Furthermore, the process as described here functions independently of the underlying individual defocus gradients in the electron-microscopic images.

\section{References}

Adrian, M., Dubochet, J., Lepault, J., McDowall, A.W., 1984. Cryoelectron microscopy of viruses. Nature 308 (5954), 32-36.

Bottcher, B., Wynne, S.A., Crowther, R.A., 1997. Determination of the fold of the core protein of hepatitis B virus by electron cryomicroscopy. Nature 386 (6620), 88-91.

Conway, J.F., Steven, A.C., 1999. Methods for reconstructing density maps of "single" particles from cryoelectron micrographs to subnanometer resolution. J. Struct. Biol. 128 (1), 106-118.

Erickson, H.P., Klug, A., 1971. Measurement and compensation of defocusing and aberrations by fourier processing of electron micrographs. Philos. Trans. R. Soc. Lond. 261, 105-118.

Fernandez, J.J., Sanjurjo, J.R., Carazo, J.M., 1997. A spectral estimation approach to contrast transfer function detection in electron microscopy. Ultramicroscopy 68, 267-295.

Frank, J., 1973. The envelope of electron microscopic transfer functions for partially coherent illumination. Optik 38 (5), 519-536.

Frank, J., Penczek, P., 1995. On the correction of the contrast transfer function in biological electron microscopy. Optik 98 (3), 125-129.

Frank, J., Van Heel, M., 1982. Correspondence analysis of aligned images of biological particles. J. Mol. Biol. 161, 134-137.

Gao, H., Spahn, C.M., Grassucci, R.A., Frank, J., 2002. An assay for local quality in cryo-electron micrographs of single particles. Ultramicroscopy 93 (2), 169-178.

Glaeser, R.M., Downing, K.H., 1992. Assessment of resolution in biological electron crystallography. Ultramicroscopy 47, 256-265.

Hewat, E.A., Neumann, E., 2002. Characterization of the performance of a $200-\mathrm{kV}$ field emission gun for cryo-electron microscopy of biological molecules. J. Struct. Biol. 139, 60-64.

Kastner, B., 1998. Purification and electron microscopy of spliceosomal snRNPs. In: Schenkel, J. (Ed.), RNP Particles, Splicing and Autoimmune Diseases, pp. 95-140.

Lebart, L., Morineau, A., Warwick, K.M., 1984. Elements of applied stochastic processes. In: Bhat, U.N. (Ed.), Multivariate Descriptive Statistical Analysis, second ed. Wiley, New York.

Ludtke, S.J., Baldwin, P.R., Chiu, W., 1999. EMAN: semiautomated software for high-resolution single-particle reconstructions. J. Struct. Biol. 128 (1), 82-97.

Radermacher, M., Ruiz, T., Wieczorek, H., Gruber, G., 2001. The structure of the V1-ATPase determined by three-dimensional electron microscopy of single particles. J. Struct. Biol. 135, 26-37.

Saad, A., Ludtke, S.J., Jakana, J., Rixon, F.J., Tsuruta, H., Chiu, W., 2001. Fourier amplitude decay of electron cryomicroscopic images 
of single particles and effects on structure determination. J. Struct. Biol. 133 (1), 32-42.

Scherzer, O., 1949. The theoretical resolution limit of the electron microscope. J. Appl. Phys. 20, 20.

Stark, H., Dube, P., Lührmann, R., Kastner, B., 2001. Arrangement of RNA and proteins in the spliceosomal U1 small nuclear ribonucleoprotein particle. Nature 409 (6819), 539-542.

Tani, K., Sasabe, H., Toyoshima, C., 1996. A set of computer programs for determining defocus and astigmatism in electron images. Ultramicroscopy 65, 31-44.

Thon, F., 1966. Zur Defokussierunsabhaengigkeit des Phasenkontrastes bei der elektronenmikroskopischen Abbildung. Z. Naturforschg. 21a, 476-478.

Tischendorf, G.W., Zeichhardt, H., Stöffler, G., 1974. Determination of the location of proteins L14, L17, L18, L19, L22 and L23 on the surface of the 50S ribosomal subunit of Escherichia coli by immune electron microscopy. Mol. Gen. Genet. 134, 187-208.

Toyoshima, C., Unwin, N., 1988. Contrast transfer for frozenhydrated specimens: determination from pairs of defocused images. Ultramicroscopy 25 (25), 279-292.

Toyoshima, C., Yonekura, K., Sasabe, H., 1993. Contrast transfer for frozen-hydrated specimens. II. Amplitude contrast at very low frequencies. Ultramicroscopy 48, 165-176.

Valentine, R.C., Shapiro, B.M., Stadtman, E.R., 1968. Regulation of glutamine synthetase. XII. Electron microscopy of the enzyme from Escherichia coli. Biochemistry 7 (6), 2143-2152.
Van Heel, M., 1984. Multivariate statistical classification of noisy images (randomly oriented biological macromolecules). Ultramicroscopy 13 (1-2), 165-183.

van Heel, M., Gowen, B., Matadeen, R., Orlova, E.V., Finn, R., Pape, T., Cohen, D., Stark, H., Schmidt, R., Schatz, M., Patwardhan, A., 2000. Single-particle electron cryo-microscopy: towards atomic resolution. Q. Rev. Biophys. 33 (4), 307-369.

van Heel, M., Harauz, G., Orlova, E.V., Schmidt, R., Schatz, M., 1996. A new generation of the IMAGIC image processing system. J. Struct. Biol. 116 (1), 17-24.

Wade, R.H., Frank, J., 1977. Electron microscope transfer functions for partially coherent axial illumination and chromatic defocus spread. Optik 49 (2), 81-92.

Zemlin, F., 1994. Expected contributions of the field-emission gun to high-resolution transmission electron microscopy. Micron 25, 223226.

Zemlin, F., Beckmann, E., Zeitler, E., 1994. Electron Microscopy, ICEM-13 Paris, France.

Zhou, Z.H., Hardt, S., Wang, B., Sherman, M.B., Jakana, J., Chiu, W., 1996. CTF determination of images of ice-embedded single particles using a graphics interface. J. Struct. Biol. 116 (1), 216-222.

Zhu, J., Penczek, P.A., Schroder, R., Frank, J., 1997. Three-dimensional reconstruction with contrast transfer function correction from energy-filtered cryoelectron micrographs: procedure and application to the 70S Escherichia coli ribosome. J. Struct. Biol. 118 (3), 197-219. 